"Operation not needed" is defined as patients who were safely discharged without an operation or patients who had an operation, but nothing was found. The distribution of data samples for prediction task 2 is 37 operation not needed cases and 38 operation of benefit cases. An experienced surgical resident from UPMC manually segmented 3D PI ROIs from the CT scans ( $5 \mathrm{~mm}$ Axial cut) for each case. The most concerning $\sim 10-15 \mathrm{~cm}$ segment of bowel for necrosis with a $1 \mathrm{~cm}$ margin was selected. A total of 7 slices per patient were segmented for consistency. For both prediction task 1 and prediction task 2, we independently completed the following procedure for testing and training: 1.) Extracted radiomic features from the 3D PI ROIs that resulted in 99 total features. 2.) Used LASSO feature selection to determine the subset of the original 99 features that are most significant for performance of the prediction task. 3.) Used leave-one-out cross-validation for testing and training to account for the small dataset size in our preliminary analysis. Implemented and trained several machine learning models (AdaBoost, SVM, and Naive Bayes). 4.) Evaluated the trained models in terms of AUC and Accuracy and determined the ideal model structure based on these performance metrics. RESULTS/ANTICIPATED RESULTS: Prediction Task 1: The top-performing model for this task was an SVM model trained using 19 features. This model had an AUC of 0.79 and an accuracy of $75 \%$. Prediction Task 2: The top-performing model for this task was an SVM model trained using 28 features. This model had an AUC of 0.74 and an accuracy of 64\%. DISCUSSION/SIGNIFICANCE OF IMPACT: To the best of our knowledge, this is the first study to use radiomic-based machine learning models for the prediction of tissue ischemia, specifically intestinal ischemia in the setting of PI. In this preliminary study, which serves as a proof of concept, the performance of our models has demonstrated the potential of machine learning based only on radiomic imaging features to have discriminative power for surgical decision-making problems. While many non-imaging-related clinical factors play a role in the gestalt of clinical decision making when PI presents, we have presented radiomic-based models that may augment this decision-making process, especially for more difficult cases when clinical features indicating acute abdomen are absent. It should be noted that prediction task 2, whether or not a patient presenting with PI would benefit from an operation, has lower performance than prediction task 1 and is also a more challenging task for physicians in real clinical environments. While our results are promising and demonstrate potential, we are currently working to increase our dataset to 300 patients to further train and assess our models. References DuBose, Joseph J., et al. "Pneumatosis Intestinalis Predictive Evaluation Study (PIPES): a multicenter epidemiologic study of the Eastern Association for the Surgery of Trauma." Journal of Trauma and Acute Care Surgery 75.1 (2013): 15-23. Knechtle, Stuart J., Andrew M. Davidoff, and Reed P. Rice. "Pneumatosis intestinalis. Surgical management and clinical outcome." Annals of Surgery 212.2 (1990): 160.

3032

\section{MKit: Pilot Results of Primary Prevention Sexual Violence WebApp}

Michelle Munro-Kramer ${ }^{1}$, Lindsay Cannon ${ }^{1}$, Jose Bauermeister ${ }^{2}$, Yasamin Kusunoki ${ }^{1}$, Quyen Ngo ${ }^{1}$ and Rob Stephenson ${ }^{1}$

${ }^{1}$ University of Michigan and ${ }^{2}$ University of Pennsylvania

OBJECTIVES/SPECIFIC AIMS: Sexual violence (SV) is a public health crisis. High rates of SV are observed among college-age youth, yet holistic interventions are currently lacking. The purpose of this study was to conduct a pilot feasibility and acceptability test of a WebApp, MKit, which translates a clinical life skills approach to influence the health and well-being of university students. METHODS/STUDY POPULATION: We randomized two residence halls at a public university in the Midwest into a control group $(n=139)$ that received typical university programming around SV and healthy relationships, or an intervention group $(n=122)$ receiving MKit and the usual SV programming. We used online surveys to assess acceptability, feasibility, and usability at 3- and 5-months. Focus groups were conducted with a subsample of participants at 5-months to further investigate safety. RESULTS/ANTICIPATED RESULTS: The mean number of uses of MKit was 2.84 in a 5-month period. The majority of intervention participants endorsed the acceptability and usability of MKit as easy to use, well integrated, accessible, and easy to learn quickly. There were no concerns regarding personal safety. DISCUSSION/SIGNIFICANCE OF IMPACT: MKit provides a promising resource platform to deliver messages regarding healthy relationships and SV within the university context. By delivering SV-related content through a holistic life skills approach, MKit may offer new opportunities to reach and engage a wide range of students on how to foster healthy relationships.

3086

\section{Virtual World-based Cardiac Rehabilitation to Promote Healthy Lifestyle Among Cardiac Patients}

LaPrincess Brewer ${ }^{1}$, Brian Kaihoi, Shawn Leth, Ray Squires,

Randal Thomas, Robert Scales, Jorge Trejo-Gutierrez and

Stephen Kopecky

${ }^{1}$ Mayo Clinic

OBJECTIVES/SPECIFIC AIMS: Our aim was to assess the feasibility and acceptability of a VW-based cardiac rehabilitation (CR) program (Destination Rehab) as an extension of a face-to-face conventional CR program. We hypothesized that a VW-based CR program could be successfully implemented as an extension of conventional $\mathrm{CR}$ and would have high acceptability among cardiac patients. METHODS/STUDY POPULATION: We recruited 30 adult cardiac patients (10/site) hospitalized at Mayo Clinic Hospitals in Rochester, $\mathrm{MN}$, Jacksonville, FL or Scottsdale, AZ with a diagnosis for CR (eg, acute coronary syndrome (ACS), heart failure, elective percutaneous coronary intervention (PCI)). Other inclusion criteria included at least 1 modifiable, lifestyle risk factor target: sedentary lifestyle ( $<3$ hours physical activity (PA)/week), unhealthy diet $(<5$ servings fruits and vegetables/day) or current smoking ( $>1$ year). Patients participated in an 8-week, health education program using a VW platform from a prior proof-of-concept study and provided intervention usability, usefulness and satisfaction feedback. We assessed cardiovascular (CV) health behaviors (diet, PA) and risk factors (eg, blood pressure (BP), lipids) at baseline and immediate postintervention. RESULTS/ANTICIPATED RESULTS: Among 30 patients enrolled (mean age; 59 years; $50 \%$ women; $65 \%<$ college graduate; $32 \%$ annual household income $<\$ 50,000$ ), 28 (98\%) completed the study. The majority (64\%) were enrolled in conventional CR with a high session completion rate (median 36 sessions, interquartile range 8-36). The most common CR indication was PCI (68\%). There were statistically significant improvements in PA from baseline to post-intervention: vigorous PA, +10.7 (SD 11.7) minutes/ day $(\mathrm{p}=0.05)$ and flexibility exercises +0.9 (SD 0.9) days/week for men $(\mathrm{p}=0.05)$. There were favorable trends in risk factors: systolic BP 